\title{
Feed Forward Neural Network Classification for INDIAN Krishna Kamod Rice
}

\author{
Niky K. Jain* \\ Research Scholar \\ RKU \& Assistant Professor \\ MSc(IT) Deptt., ISTAR, \\ V.V.Nagar, Anand
}

\author{
Samrat O. Khanna \\ Professor \& Head, \\ MSc(IT) Deptt., \\ ISTAR, V.V.Nagar, \\ Anand
}

\author{
Maheshwari Chetna V. \\ Assistant Professor \\ EC Deptt., G H Patel \\ College of Engg \& Technology, \\ V.V.Nagar, Anand
}

\begin{abstract}
The Carrying out effective and sustainable agriculture product has become an important issue in recent years. Agricultural production has to keep up with an ever-increasing population. A key to this is the usage of modern techniques (for precision agriculture) to take advantage of the quality in the market. Classification of rice seeds from the bare human hands is neither cost effective nor recommended. The automatic grading for analysis of quality has become the need of the hour. This paper recommends an add-on approach to quality experts for the quality analysis of INDIAN Krishna Kamod Rice using computer vision and soft computing techniques. Computer Vision provides a grading methodology, nondestructive technique, along with multi-layer feed forward neural networking which achieves high degree of quality than human vision inspection.
\end{abstract}

\section{Keywords}

Computer Vision, feed forward neural network, Indian Krishna Kamod rice seeds, non-destructive.

\section{INTRODUCTION}

Indian agricultural production and development is considered to be too oldest and most widespread in the whole world. In this era of Digital India where hi-tech upbringing of an agricultural industry has become more intellectual and automatic machinery has replaced the human efforts [1]. In India it is high time to overcome the need of ever-increasing demand of production by advancement in agricultural sector. Due to automation need of high quality and safety standards achieved with accuracy, along with fast and cost effective quality determination of agricultural products has increased a lot [11]. Quality being a major consideration in any food product is determined from its physiochemical properties by human sensory panel which is time consuming, may be varying results and costly as well as destructive chemical analysis [10]. Significant developments have been made in this field of computer vision since past few years [4]. Efforts are being geared towards the replacement of traditional human sensory panel with automated systems, as human operations are inconsistent and less efficient [5].

Oryza Sativa L. (Rice) is a vital worldwide agriculture product. It is one of the leading food crops of the world as more than half of the world's population relies on rice as the major daily source of calories and protein. Rice (Oryza Sativa L) is cultivated in several countries such as India, China, Indonesia, Bangladesh and Thailand which are considered as the major producers. India is the world's $2^{\text {nd }}$ largest producer and consumer country of rice for a very long time. It is one of the leading food crops of the world as more than half of the world's population relies on rice as the major daily source of calories and protein.
The research work summarized in this paper focuses on the problem faced by Indian Rice industry and its cost effective solution. Second section comprises of the particular problem of quality evaluation of Krishna Kamod Rice seeds. The next section discusses about the various proposed methodology being used along with the materials for calculating parameters for the quality of rice seeds. The proposed system as well as proposed algorithm for computing Rice seeds with long, small and normal seeds classification is also discussed in the same section. Section 4 concentrates on the quantification for the quality of rice seeds based on computer vision processing and analysis. Section 5 shows classification of rice seeds using multi-layer feed forward neural network for quality evaluation. Section 6 provides the conclusion of the proposed process.

\section{PROBLEM DEFINITION}

Krishna Kamod rice (Oryza Sativa L) seeds contain foreign elements in terms of long as well as small seed as shown in Figure 1. These seeds are having very much importance in quantifying quality. At the time of processing these seeds are removed.

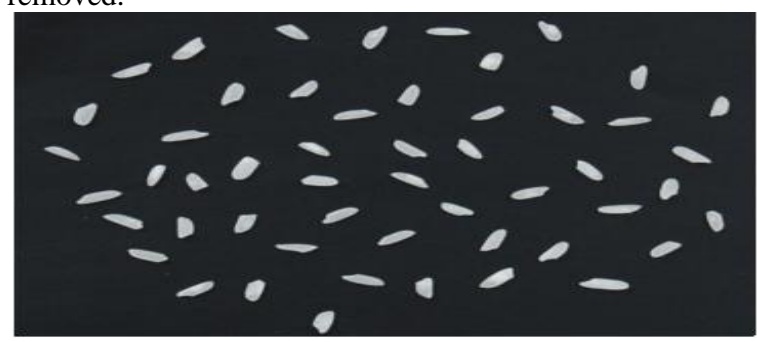

Fig. 1 Rice seeds with and without foreign elements
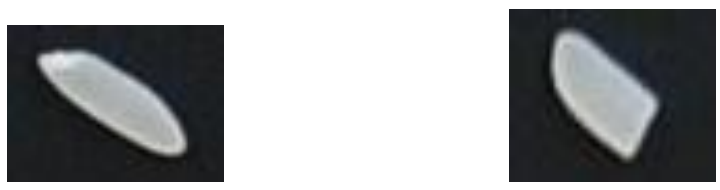

Fig. 2 Foreign elements in the sample

Proper removal of this seed is necessary if it is not so then it creates degradation in quality of rice seed. This paper proposes a new method for counting the number of Krishna Kamod rice (Oryza Sativa L) seeds with these foreign elements as shown in Figure 2 using non-destructive technique based on artificial neural network to quantify the quality of Krishna Kamod rice (Oryza Sativa L) seeds.

\section{MATERIALS AND METHODS}

In this section we discuss the proposed algorithm. Here we have used different varietal samples of Krishna Kamod rice. We define quality based on the combined measurement technique. We use minor axis length, major axis length, 
eccentricity and area of rice seed for counting the number of Krishna Kamod rice (Oryza sativa L) seeds with long seeds, normal seeds as well as small seeds.

\subsection{System Description and Operating Procedure}

A schematic diagram of the proposed system is in Figure 3.In our proposed system there is a camera which is mounted on the top of the box at point 1 in Figure 3. The camera is having 12 mega pixels quality with8X optical zoom. After capturing images of rice seed by camera is stored for further processing.

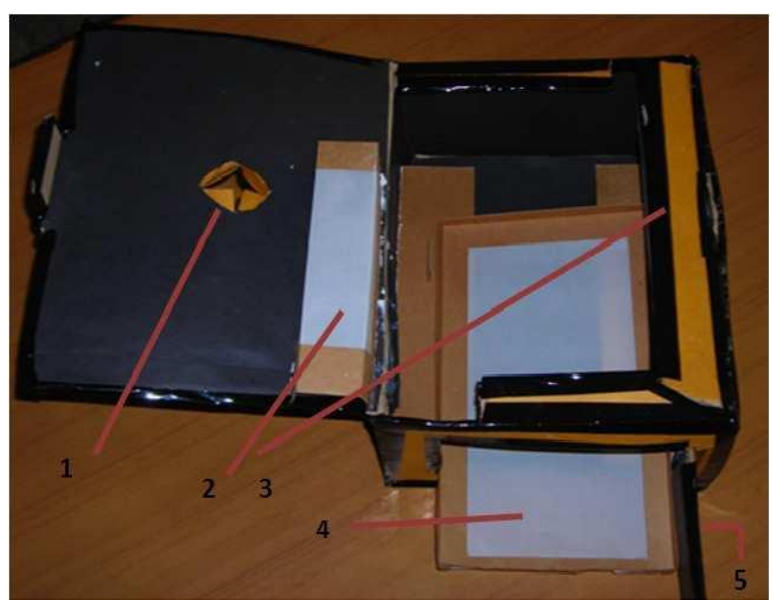

Fig. 3 Proposed Computer vision System for Analysis of Rice Seeds

The simplicity of operation of system can be concluded from the operating procedure detailed in Table 1 .

Table 1. Operating Procedure for Proposed System

\begin{tabular}{|l|l|}
\hline Sr. No. & STEPS \\
\hline $\mathbf{1}$ & $\begin{array}{l}\text { Uniformly spread the sample of seeds on the tray } \\
\text { without overlapping each other. }\end{array}$ \\
\hline $\mathbf{2}$ & Capture image of seeds form the system \\
\hline $\mathbf{3}$ & Processing and analysis of captured image \\
\hline $\mathbf{4}$ & $\begin{array}{l}\text { Display number of normal rice seeds, long seeds } \\
\text { and small seeds. }\end{array}$ \\
\hline $\mathbf{5}$ & Repeat above steps for 10 to 15 samples \\
\hline
\end{tabular}

\subsection{Proposed algorithm to detect rice seeds with long and small seeds}

According to our proposed algorithm first capture image of sample spread on the black or butter paper using camera.

Table 2. Proposed Algorithm

\begin{tabular}{|l|l|}
\hline Sr. No. & STEPS \\
\hline $\mathbf{1}$ & Select the region of interest of the rice seeds \\
\hline $\mathbf{2}$ & Convert the RGB image to grey images \\
\hline $\mathbf{3}$ & Apply the edge detection operation \\
\hline $\mathbf{4}$ & Calculate the parameters of the rice seeds \\
\hline
\end{tabular}

\begin{tabular}{|l|l|}
\hline $\mathbf{5}$ & $\begin{array}{l}\text { Compute the histogram of the parameters of rice } \\
\text { seeds and find out the threshold ranges. }\end{array}$ \\
\hline $\mathbf{6}$ & $\begin{array}{l}\text { Train the soft computing technique for classifying } \\
\text { normal, long and small rice seeds. }\end{array}$ \\
\hline $\mathbf{7}$ & $\begin{array}{l}\text { Test Rice seeds of unknown sample for counting } \\
\text { normal, long and small rice seeds. }\end{array}$ \\
\hline
\end{tabular}

This image is color image so we covert it in to grey scale image as the color information is not of importance. The identification of objects within an image is a very difficult task. One way to make straightforward the problem is to use optimal edge detector, ISEF [10], for extracting edges of grey scale image. This phase identifies individual object boundaries and marks the Centre of each object for further processing. Thresholding is used to convert the segmented image to a binary image. The output binary image has value 1 (White areas) for all pixels of edges and 0 (black) for all other pixels.

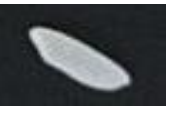

(a)

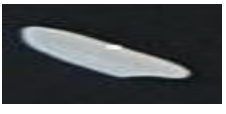

(b)

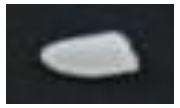

(c)
Fig. 4 Rice seed with and without foreign elements

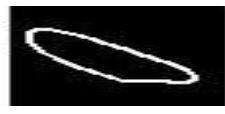

(a)

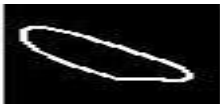

(b)

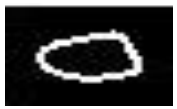

(c)
Fig. 5 After edge detection operation rice seed without and with foreign elements

In Figure 4(a) normal rice seed of good quality is shown, while Figure 4(b) and Figure 4(c) contains an image of a long seed and small seed. After applying the Edge detection operation, we get images of Figures 5(a), (b) and (c) respectively.

\subsection{Parameter Calculation}

Here we are extracting four parameters minor axis length, major axis length, eccentricity and area for differentiating normal rice seed from long seed as well as small seed.

"The minor axis length $\mathrm{M}$ of an image is defined as the length (in pixels) of the minor axis of the ellipse that has the same normalized second central moments as the region."

"The major axis length $\mathrm{N}$ of an image is defined as the length (in pixels) of the major axis of the ellipse that has the same normalized second central moments as the region."

"The eccentricity $\mathrm{E}$ is the ratio of the distance between the foci of the ellipse and its major axis length. The value is between 0 and 1."

"The area A of any object in an image is defined by the total number of pixels enclosed by the boundary of the object." 


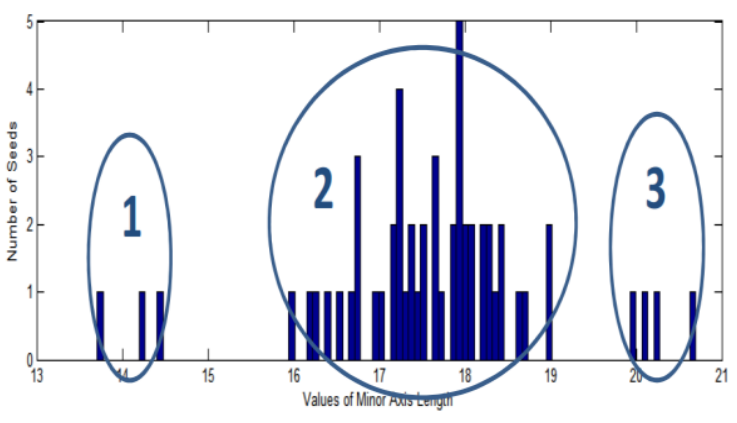

Fig. 6 (a) Histogram for Minor axis length

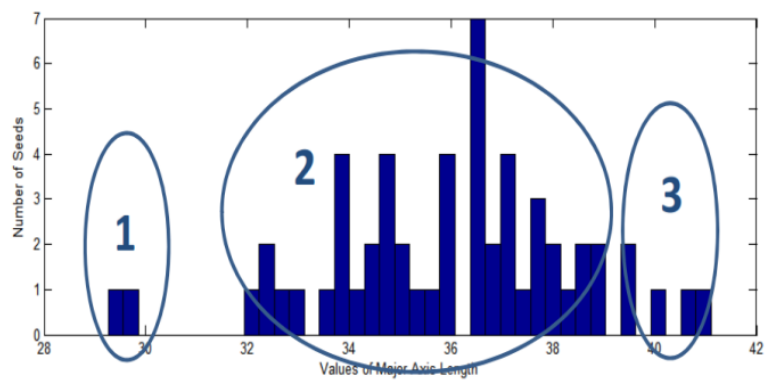

Fig. 6 (b) Histogram for Major axis length

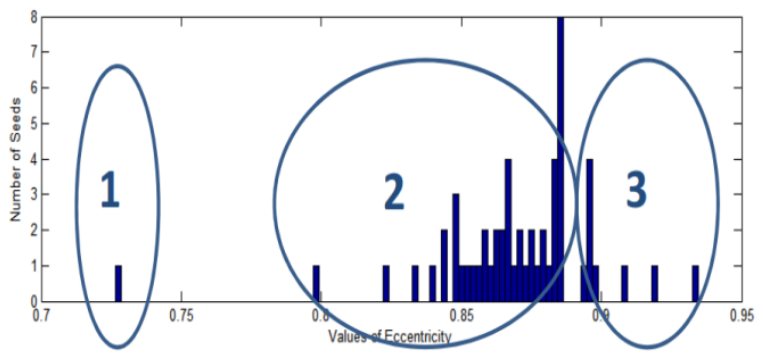

Fig. 6 (c) Histogram for Eccentricity

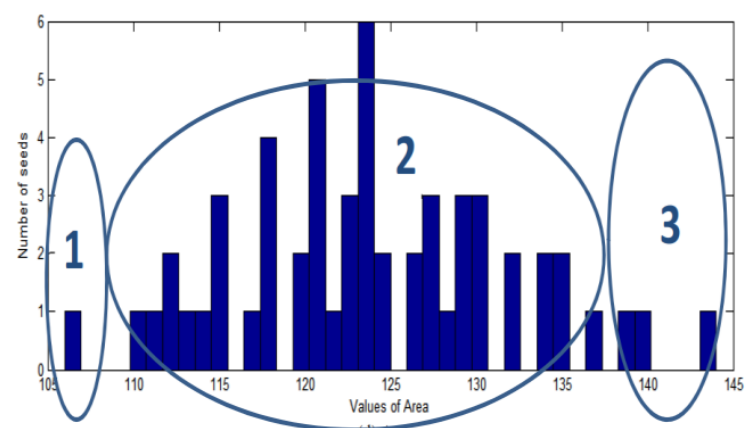

Fig. 6 (d) Histogram for Area

For minor axis calculation, we define minor axis of a small seed as $\mathbf{1}$, minor axis of normal seed as $\mathbf{2}$ and minor axis of large seed as $\mathbf{3}$. Minor axis $\mathbf{1}$ is having a normally less value then minor axis $\mathbf{2}$ and minor axis $\mathbf{2}$ is having a less value than minor axis 3. For eccentricity calculation a long seed is having bigger value than the normal seed and small seed. Use of Diagrams of histogram for Minor axis length, Major axis length, Eccentricity and Area calculation computed from sample is shown in Figure $6(\mathrm{a}-\mathrm{d})$.

\section{RESULT ANALYSIS}

Table 3 shows intended parameters value based on histogram for normal seeds, long seeds and small seeds respectively. It shows values of Minor axis length (MN), Major axis length (MJ), Eccentricity (E) and Area (A) of various rice seeds available in one sample. Same way values of all four parameters of 15 samples are found.

Table 3 Analysis for Several Seeds Available In One Sample

\begin{tabular}{|c|c|c|c|c|c|c|c|c|c|}
\hline S.No. & MN & \multicolumn{2}{c}{ MJ } & E & A & S.No. & MN & MJ & E \\
\hline 1 & 18.65884 & 39.97138 & 0.88436 & 140 & 11 & 18.0689 & 34.8726 & 0.855296 & 121 \\
\hline 2 & 18.34864 & 35.8962 & 0.859487 & 121 & 12 & 17.02302 & 36.62994 & 0.885453 & 129 \\
\hline 3 & 17.84476 & 36.49475 & 0.872302 & 127 & 13 & 16.28225 & 33.88045 & 0.876951 & 115 \\
\hline 4 & 17.16051 & 36.68574 & 0.88385 & 124 & 14 & 16.96713 & 33.04083 & 0.858078 & 112 \\
\hline 5 & 17.97317 & 37.00356 & 0.874117 & 126 & 15 & 14.44732 & 40.57272 & 0.934454 & 139 \\
\hline 6 & 20.1138 & 29.26412 & 0.726355 & 106 & 16 & 16.52442 & 36.99952 & 0.894728 & 130 \\
\hline 7 & 18.40508 & 34.74481 & 0.848172 & 118 & 17 & 18.01508 & 38.66314 & 0.884811 & 124 \\
\hline 8 & 18.23306 & 39.34479 & 0.88614 & 135 & 18 & 17.38939 & 37.43574 & 0.885566 & 135 \\
\hline 9 & 15.95813 & 38.38183 & 0.909468 & 130 & 19 & 20.24039 & 41.11323 & 0.870421 & 144 \\
\hline 10 & 17.44217 & 37.63879 & 0.886144 & 125 & 20 & 17.92548 & 29.73881 & 0.79792 & 111 \\
\hline
\end{tabular}

For finding out the number of normal rice seeds, long rice seeds and small rice seeds we compute thresholds values using the histograms of Figure $6(\mathrm{a}-\mathrm{d})$ for minor axis length, major-axis length, eccentricity and area as mention in Table 4. 
Table 4 Computed Threshold Values

\begin{tabular}{|c|c|c|c|}
\hline Parameters & Small seed & $\begin{array}{c}\text { Normal } \\
\text { seed }\end{array}$ & $\begin{array}{c}\text { Long } \\
\text { seed }\end{array}$ \\
\hline $\begin{array}{c}\text { Minor Axis } \\
\text { Length }\end{array}$ & $11-15$ & $15-20$ & $20-25$ \\
\hline $\begin{array}{c}\text { Major Axis } \\
\text { Length }\end{array}$ & $20-28$ & $28-37$ & $40-47$ \\
\hline Eccentricity & $0.67-0.82$ & $0.82-0.92$ & $0.92-0.98$ \\
\hline Area & $100-110$ & $110-160$ & $160-180$ \\
\hline
\end{tabular}

\section{CLASSIFICATION}

\subsection{Neural Network (NN):}

A two layer feed forward neural network with 5 nodes in each layer is used for classification. The training function using gradient descent with momentum weight as learning function, and maximum likelihood as a performance function is used to train the NN. Here in figure $7 \mathrm{Gmn}, \mathrm{Gmj}$, Gecc and Gar are grades of Minor axis length, Major axis length, Eccentricity and Area respectively. N11, N12, N13, N14 and N15 are nodes in Hidden layer 1 while N21, N22, N23, N24 and N25 are nodes in Hidden layer 2. Output labels are 111, 222 and 333. 111 is assigned for small seed, 222 for normal and 333 for large seed.

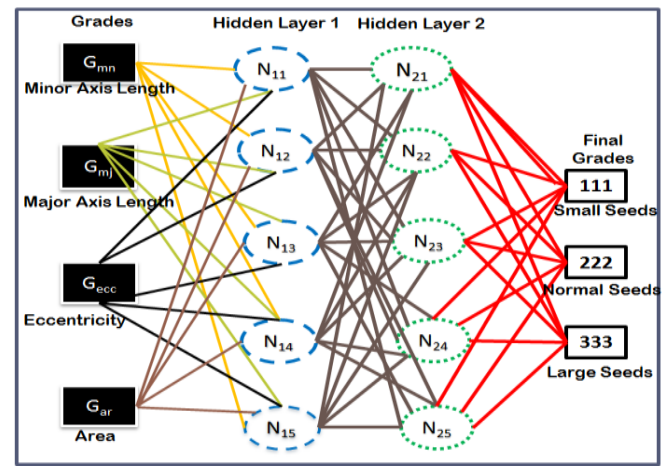

Fig. 7 Schematic diagram of trained two layer feed forward neural network of Krishna Kamod rice seeds of 15 samples

Table 4 Grading Of Several Seeds Based On Priority of Parameters Available In One Sample

\begin{tabular}{|c|c|c|c|c|c|c|c|c|c|}
\hline S.No. & $\mathbf{m n}$ & $\mathbf{G}_{\mathbf{m n}}$ & $\mathbf{m j}$ & $\mathbf{G}_{\mathbf{m j}}$ & $\mathbf{e c c}$ & $\mathbf{G}_{\mathbf{e c c}}$ & $\mathbf{a r}$ & $\mathbf{G}_{\mathbf{a r}}$ & $\mathbf{G r a d e}$ \\
\hline 1 & 18.65884 & 2 & 39.97138 & 2 & 0.88436 & 2 & 140 & 2 & 222 \\
\hline 2 & 18.34864 & 2 & 35.8962 & 2 & 0.859487 & 2 & 121 & 2 & 222 \\
\hline 3 & 17.84476 & 2 & 36.49475 & 2 & 0.872302 & 2 & 127 & 2 & 222 \\
\hline 4 & 17.16051 & 2 & 36.68574 & 2 & 0.88385 & 2 & 124 & 2 & 222 \\
\hline 5 & 17.97317 & 2 & 37.00356 & 2 & 0.874117 & 2 & 126 & 2 & 222 \\
\hline
\end{tabular}


Table 6 Parameters Used For Neural Network In XL Miner

\begin{tabular}{|l|l|}
\hline \multicolumn{2}{|c|}{ Parameters / Options } \\
\hline Cost Function & Maximum Likelihood \\
\hline Hidden Layer Sigmoid & Standard \\
\hline Output Layer Sigmoid & Standard \\
\hline Epochs & 30 \\
\hline Step size for Gradient descent & 0.1 \\
\hline Weight change momentum & 0.6 \\
\hline Error Tolerance & 0.01 \\
\hline
\end{tabular}

When the neural network training was finished, the network was tested with unknown sample. The classification accuracy of test dataset is $97 \%$.

\section{CONCLUSION}

Quality quantification of Krishna Kamod rice seeds using computer vision analysis and soft computing techniques have

given an advancement in the agricultural sector of India. Here we were able to calculate geometrical features like minor axis length, major axis length, eccentricity and area for counting normal seed and foreign element in terms of long as well as small seed for a given sample. A new technology with nondestructive quality analysis of rice seeds using feed forward neural network provides an accuracy of $97 \%$ and was quiet effectively used in the rice mills of nearby areas. Traditionally quality evaluation and assessment is done by human sensory panel which is time consuming and costly.

\section{ACKNOWLEGMENT}

The author expresses their gratitude to Mr. H. N. Shah the owner of Shri Krishna Rice and Pulse Mill, Borsad for the system model as per the requirements desired by the image processing group. We also convey thanks to Mr. A. B. Patel and Mr.V.B.Patel from the Chandan Rice and Pulse Mill GIDC, Khambhat for their helpful advice.

\section{REFERENCES}

[1] Abdullah MZ, Fathinul-Syahir AS, Mohd-Azemi BMN, "Automated inspection system for color and shape grading of star fruit (Averrhoa carambola L.) using
Computer vision sensor," Transactions of the Institute of Measurement and Control, 27 (2), 65-87, 2005.

[2] Abutaleb AS, "Automatic thresholding of grey-level pictures using two-dimensional entropies." Pattern Recognition, 47(1), 22-32, 1989.

[3] Ballard, D. A., \& Brown, C. M. " Computer vision", Englewood Cliffs, NJ, USA: Prentice-Hall ,1982.

[4] Blasco J, Aleixos N, Molt E, "Computer vision system for automatic quality grading of fruit", Biosystems Engineering, 415-423, 2003.

[5] Chetna Maheshwari, Kavindra Jain, Chintan Modi, "Novel approach for Oryza sativa L.(Rice) based on Computer vision technology," PEPCCI, National Conference ,ISBN No.-978-93-81286-06-7,2012.

[6] Du CJ, Sun D-W, "Recent development in the applications of image processing techniques for food quality evaluation." Trends in Food Science and Technology, 15,230-249, 2004.

[7] Du C-J, Sun D-W, "Learning techniques used in computer vision for food quality evaluation: a review", Journal of Food Engineering, 72(1), 39-55, 2006.

[8] Gunasekaran Sundaram, Kexiang Ding, "Computer vision technology for food quality assurance,". Trends in Food Science and Technology, 7, 245-256, 1996.

[9] Jain AK, "Fundamentals of Digital Image Processing," Englewood Cliffs: Prentice-Hall 1989.

[10] Kavindra Jain, Chintan K. Modi, Kunal Pithadiya, "Non Destructive quality evaluation in spice industry with specific reference to Cuminum Cyminum L (Cumin) seeds," International Conference on Innovations \& Industrial Applications, Malaysia, (IEEE) 2009.

[11] M. Kurita and N. Kondo, "Agricultural product grading method by image processing (part 1) - effectiveness of direct lighting method", J.SHITA 18(1): 9-17,2006.

[12] Shen Castan, Sian Zhao,"A Comparitive study of Performance of Noisy roof edge detection", 5th International conference on Computer analysis of Images and Patterns, volu.179, pp 170-174

[13] Tadhg Brosnan, Da-Wen Sun, "Improving quality inspection of food products by computer vision-a review", Journal of Food Engineering 61, pp. 3-16, 2004.

[14] Xiaopei Hu, ParmeshwaraK.M, DavidV. "Development of Non Destructive Methods To Evaluate Oyster Quality By Electronic Nose Technology", Springer Science Business Media, LLC, 2008. 